PPD TNF

\begin{tabular}{|c|c|c|c|c|}
\hline MEDIUM & $\begin{array}{l}\text { MEDIUM } \\
\text { INFLIX }\end{array}$ & ANTIGEN & $\begin{array}{l}\text { ANTIGEN } \\
\text { INFLIX }\end{array}$ & BEAD \\
\hline $\begin{array}{c}175.611 \\
391.512 \\
70.095 \\
50.787 \\
155.334 \\
\end{array}$ & $\begin{array}{c}129.126 \\
\\
38.901 \\
44.073 \\
0 \\
0.291 \\
8.667 \\
\end{array}$ & $\begin{array}{c}480.249 \\
364.134 \\
186.072 \\
334.095 \\
37.287 \\
31.98 \\
166.92 \\
\end{array}$ & $\begin{array}{c}41.898 \\
\\
34.134 \\
22.107 \\
47.229 \\
0.291 \\
11.979 \\
35.271 \\
\end{array}$ & $\begin{array}{c}386.404 \\
421.329 \\
16.45 \\
\\
12.366 \\
19.14 \\
23.614 \\
191.967 \\
\\
336.303 \\
110.262 \\
95.478 \\
122.841 \\
518.088 \\
96.831 \\
386.404 \\
\end{array}$ \\
\hline
\end{tabular}

\title{
Treated TNF
}

\begin{tabular}{|c|c|c|c|c|}
\hline MEDIUM & $\begin{array}{l}\text { MEDIUM } \\
\text { INFLIX }\end{array}$ & ANTIGEN & $\begin{array}{l}\text { ANTIGEN } \\
\text { INFLIX }\end{array}$ & BEAD \\
\hline & & & & 168.822 \\
& & & & 288.834 \\
& & & & 147.246 \\
& & & & 67.671 \\
& & & & 106.128 \\
& & & & 125.565 \\
& & & & 82.026 \\
113.778 & 99.588 & 420.264 & 128.331 & 80.511 \\
142.701 & 43.047 & 0 & 0 & 273.805 \\
379.338 & 14.481 & 29.508 & 0 & 463.608 \\
181.188 & 115.449 & 68.64 & 71.565 & 382.009 \\
126.135 & 68.64 & & & \\
192.183 & 71.565 & 99.15 & 75.51 & 719.406 \\
127.848 & 67.671 & 68.64 & 65.265 & 62.477 \\
87.126 & 61.923 & & & 39.599 \\
24.033 & 0 & 39.068 & 27.249 & 44.022 \\
58.271 & 28.177 & 65.015 & 57.228 & 79.889
\end{tabular}




\begin{tabular}{|c|c|c|c|c|}
16.415 & 0 & 36.959 & 28.642 & 47.002 \\
70.775 & 22.934 & 74.658 & 3.687 & 568.822 \\
48.218 & 14.538 & 105.593 & 0 & 144.022 \\
213.778 & 199.588 & 320.264 & 228.331 & 239.599 \\
46.415 & 2.36 & 66.959 & 28.642 & 653.312 \\
58.271 & 28.177 & 145.015 & 57.228 & 247.002 \\
148.218 & 84.538 & 905.593 & 139.36 & 273.805 \\
\hline
\end{tabular}

Active disease TNF

\begin{tabular}{|c|c|c|c|c|}
\hline MEDIUM & $\begin{array}{l}\text { MEDIUM } \\
\text { INFLIX }\end{array}$ & ANTIGEN & $\begin{array}{l}\text { ANTIGEN } \\
\text { INFLIX }\end{array}$ & BEAD \\
\hline $\begin{array}{c}291.078 \\
1.323 .861 \\
34.134 \\
140.649 \\
63.054 \\
45.108 \\
390.111 \\
189.738 \\
399.348 \\
20.414 \\
9.068 \\
292.191 \\
1159.93 \\
102.354 \\
19.164 \\
6.075 \\
391.078 \\
129.068 \\
940.649 \\
1.323 .861 \\
845.108\end{array}$ & $\begin{array}{c}269.007 \\
68.118 \\
29.835 \\
68.049 \\
38.484 \\
33.054 \\
203.421 \\
11.475 \\
101.853 \\
6.122 \\
7.8 \\
1.035 \\
33.298 \\
0 \\
0 \\
0 \\
269.007 \\
87.8 \\
268.049 \\
768.118 \\
233.054\end{array}$ & $\begin{array}{c}2.348 .133 \\
99.87 \\
149.067 \\
135.108 \\
92.304 \\
118.482 \\
0 \\
20.49 \\
44.022 \\
914.239 \\
475.918 \\
122.553 \\
2.829 \\
8.245 \\
148.085 \\
914.239 \\
1.249 .067 \\
2.348 .133 \\
1.292 .304\end{array}$ & $\begin{array}{c}1.242 .699 \\
68.049 \\
115.26 \\
123.15 \\
84.888 \\
89.772 \\
0 \\
0 \\
15.098 \\
11.201 \\
250.876 \\
45.185 \\
0 \\
0 \\
0 \\
211.201 \\
515.26 \\
1.242 .699 \\
784.888\end{array}$ & $\begin{array}{c}173.508 \\
620.202 \\
59.217 \\
304.107 \\
37.392 \\
87.564 \\
407.052 \\
188.217 \\
294.954 \\
1.062 .617 \\
265.602 \\
928.983 \\
463.511 \\
74.064 \\
468.441 \\
679.681 \\
356.476 \\
24.226 \\
122.333 \\
260.568 \\
910.12 \\
26.875 \\
0.123 \\
10.078 \\
273.508 \\
122.333 \\
304.107 \\
1.620 .202 \\
787.564 \\
474.064 \\
463.511\end{array}$ \\
\hline
\end{tabular}




\begin{tabular}{|c|c|c|c|c|}
\hline MEDIUM & $\begin{array}{l}\text { MEDIUM } \\
\text { INFLIX }\end{array}$ & ANTIGEN & $\begin{array}{l}\text { ANTIGEN } \\
\text { INFLIX }\end{array}$ & BEAD \\
\hline & & & & 230.163 \\
& & & & 132.253 \\
& & & & 202.973 \\
& & & & \\
& & & & 25.36 \\
& & & & 47.956 \\
0 & 0 & 0 & 0 & 12.367 \\
525.65 & 241.156 & 867.937 & 85.415 & 0 \\
& & 389.901 & 123.402 & 280.508 \\
20.844 & 49.716 & 38.091 & 23.703 & 164.559 \\
0 & 0 & 79.215 & 76.236 & 569.326 \\
330.816 & 88.188 & 0 & 604.761 & 165.147 \\
203.478 & 67.341 & 61.44 & 91.191 & 293.025 \\
127.719 & 935.088 & 474.633 & 70.299 & 55.566 \\
& & & & 230.163 \\
\hline
\end{tabular}

Treated IFN

\begin{tabular}{|c|c|c|c|c|}
\hline MEDIUM & $\begin{array}{l}\text { MEDIUM } \\
\text { INFLIX }\end{array}$ & ANTIGEN & $\begin{array}{l}\text { ANTIGEN } \\
\text { INFLIX }\end{array}$ & BEAD \\
\hline & & & & 433.373 \\
& & & & 664.926 \\
& & & & 546.471 \\
& & & & 152.412 \\
& & & & 110.403 \\
& & & & 142.503 \\
& & & & 88.269 \\
431.879 & 323.909 & 1.628 .866 & 227.81 & 68.655 \\
1.551 .633 & 311.706 & 120.789 & 69.708 & 20.871 \\
691.143 & 0 & 105.705 & 63.096 & 31.511 \\
218.421 & 141.267 & & & 0 \\
101.784 & 62.538 & & & 10.091 \\
78.453 & 66.207 & 952.026 & 122.73 & 0 \\
82.131 & 60.093 & 182.223 & 46.659 & 21.58 \\
52.761 & 64.983 & & & 27.155 \\
34.777 & 18.555 & 30.971 & 83.868 & 24.308 \\
28.378 & 16.961 & 125.803 & 19.947 & 22.948 \\
37.982 & 17.513 & 56.379 & 17.585 & 42.188 \\
& & & &
\end{tabular}




\begin{tabular}{|c|c|c|c|c|}
65.738 & 29.076 & 468.307 & 460.646 & 333.373 \\
683.706 & 30.368 & 845.979 & 42.318 & 22.948 \\
531.879 & 323.909 & 1.328 .866 & 527.81 & 24.308 \\
37.982 & 17.513 & 56.379 & 17.585 & 321.58 \\
28.378 & 16.961 & 125.803 & 19.947 & 424.248 \\
683.706 & 130.368 & 845.979 & 242.318 & 90.871 \\
\hline
\end{tabular}

Active disease IFN

\begin{tabular}{|c|c|c|c|c|}
\hline MEDIUM & MEDIUM & ANTIGEN & $\begin{array}{c}\text { ANTIGEN } \\
\text { INFLIX }\end{array}$ & BEAD \\
& INFLIX & & & 0 \\
& & & & 0 \\
& & & & 0 \\
& & & & 66.729 \\
& & & & 553.428 \\
& & & & \\
& & & & 843.33 \\
& & & & 899.523 \\
118.285 & 0 & & & 747.822 \\
1.950 .702 & 1.626 .195 & 2.204 .711 & 1209.8 & 67.548 \\
97.898 & 0 & 143.686 & 0 & 103.211 \\
258.627 & 211.638 & 4531.53 & 1.309 .257 & 14.055 \\
1.090 .575 & 54.861 & 1.828 .992 & 1.030 .419 & 79.999 \\
1.207 .134 & 143.052 & 2.145 .084 & 389.901 & 289.175 \\
1032.81 & 581.649 & 572.859 & 293.868 & 85.804 \\
660.552 & 253.668 & 594.822 & 51.39 & 17.656 \\
1067.04 & 119.055 & 69.071 & 0 & 18.097 \\
22.446 & 15.831 & 76.187 & 36.125 & 33.42 \\
18.712 & 15.545 & 334.29 & 19.947 & 33.577 \\
704.95 & 112.468 & 105.661 & 41.906 & 212.369 \\
418.788 & 28 & 122.259 & 47.121 & 186.152 \\
701.69 & 229.744 & 929.892 & 69.094 & 747.612 \\
513.713 & 72.261 & 1.097 .087 & 94.546 & 136.369 \\
812.372 & 421.362 & 1.443 .055 & 176.28 & 218.097 \\
118.285 & 0 & 369.078 & 125.326 & 366.729 \\
118.712 & 85.545 & 334.29 & 119.947 & 893.369 \\
258.627 & 211.638 & 4531.53 & 1.309 .257 & 1375.23 \\
1.950 .702 & 1.626 .195 & 2.204 .711 & 1209.8 & 149.899 \\
1.207 .134 & 143.052 & 2.145 .084 & 389.901 & 77.116 \\
\hline & & & &
\end{tabular}




\begin{tabular}{|c|c|c|c|c|}
\hline MEDIUM & $\begin{array}{l}\text { MEDIUM } \\
\text { INFLIX }\end{array}$ & ANTIGEN & $\begin{array}{l}\text { ANTIGEN } \\
\text { INFLIX }\end{array}$ & BEAD \\
\hline & & & & 240.291 \\
& & & & 2.217 .588 \\
& & & & 1.065 .885 \\
& & & & 62.991 \\
& & & & \\
& & & & 25.364 \\
& & & & 67.514 \\
& & & & 22.747 \\
1.001 .178 & 621.063 & 1.864 .272 & 1.493 .211 & 1.435 .296 \\
& & 3.480 .225 & 871.077 & 346.132 \\
63.21 & 22.272 & 330.156 & 0 & 3.484 \\
300.303 & 81.336 & 689.529 & 133.194 & 695.358 \\
105.984 & 75.897 & 360.078 & 30.15 & 97.272 \\
59.814 & 9.249 & 453.327 & 0 & 635.466 \\
718.449 & 371.949 & 290.412 & 63.663 & 446.337 \\
& & & & 640.291 \\
\hline
\end{tabular}

Treated IL-10

\begin{tabular}{|c|c|c|c|c|}
\hline MEDIUM & $\begin{array}{l}\text { MEDIUM } \\
\text { INFLIX }\end{array}$ & ANTIGEN & $\begin{array}{l}\text { ANTIGEN } \\
\text { INFLIX }\end{array}$ & BEAD \\
\hline & & & & 3.569 .826 \\
& & & & 55.686 \\
& & & & 57.621 \\
& & & & 91.84 \\
& & & & 23.369 \\
& & & & 91.84 \\
& & & & 0 \\
2.636 .364 & 2403.93 & 2.533 .029 & 1.516 .527 & 1.957 \\
36.489 & 18.453 & 46.566 & 18.453 & 35.321 \\
67.314 & 19.398 & 32.676 & 9.981 & 32.053 \\
94.588 & 128.934 & & & 62.703 \\
0 & 0 & & & 5.698 \\
322.646 & 131.682 & 236.094 & 215.486 & 617.105 \\
28.06 & 0 & 51.415 & 19.13 & 54.31 \\
28.06 & 0 & & & 48.616 \\
69.554 & 37.11 & 61.942 & 167.736 & 45.896 \\
56.756 & 33.922 & 251.606 & 39.894 & 106.376 \\
75.964 & 35.026 & 112.758 & 35.17 & 848.496 \\
& & & &
\end{tabular}




\begin{tabular}{|c|c|c|c|c|}
131.476 & 58.152 & 936.614 & 921.292 & 2.569 .826 \\
100.253 & 46.712 & 1.691 .958 & 84.636 & 925.896 \\
2.636 .364 & 2403.93 & 1.533 .029 & 816.527 & 148.616 \\
175.964 & 135.026 & 512.758 & 235.17 & 450.127 \\
156.756 & 58.922 & 351.606 & 99.894 & 848.496 \\
100.253 & 46.712 & 1.691 .958 & 384.636 & 135.321 \\
\hline
\end{tabular}

Active IL-10

\begin{tabular}{|c|c|c|c|c|}
\hline MEDIUM & $\begin{array}{l}\text { MEDIUM } \\
\text { INFLIX }\end{array}$ & ANTIGEN & $\begin{array}{l}\text { ANTIGEN } \\
\text { INFLIX }\end{array}$ & BEAD \\
\hline & & & & $\begin{array}{c}1.586 .994 \\
866.175 \\
1672.44 \\
0 \\
0 \\
0 \\
50.868 \\
53.757 \\
74.13 \\
898.802\end{array}$ \\
\hline 1.730 .244 & 1.253 .571 & 1412.28 & 854.388 & 109.71 \\
\hline 1.864 .272 & 812.994 & 3.101 .256 & 1.423 .776 & 697.978 \\
\hline 1.966 .218 & 923.265 & 1.714 .155 & 1.579 .224 & 7.116 \\
\hline 284.151 & 32.214 & 398.043 & 157.443 & 934.464 \\
\hline 182.964 & 50.763 & 1.067 .538 & 0 & 908.857 \\
\hline 0 & 0 & 2.442 & 0 & 124.15 \\
\hline 86.865 & 55.686 & 112.614 & 100.686 & 434.387 \\
\hline 78.039 & 26.973 & 49.398 & 25.074 & 35.312 \\
\hline 60.522 & 44.142 & 53.757 & 46.059 & 36.194 \\
\hline 44.892 & 31.662 & 152.374 & 72.25 & 66.84 \\
\hline 37.424 & 31.09 & 668.58 & 39.894 & 67.154 \\
\hline 1409.9 & 224.936 & 211.322 & 83.812 & 424.738 \\
\hline 837.576 & 56 & 244.518 & 94.242 & 372.304 \\
\hline 1403.38 & 459.488 & 1.859 .784 & 138.188 & 1.495 .224 \\
\hline 1.027 .426 & 144.522 & 2.194 .174 & 189.092 & 1.586 .994 \\
\hline 1.624 .744 & 842.724 & 2886.11 & 352.56 & 536.194 \\
\hline 1.730 .244 & 1.253 .571 & 1412.28 & 854.388 & 236.359 \\
\hline 237.424 & 131.09 & 1668.58 & 839.894 & 1.866 .175 \\
\hline 384.151 & 132.214 & 698.043 & 357.443 & 550.868 \\
\hline 1.864 .272 & 812.994 & 3.101 .256 & 1.423 .776 & 934.464 \\
\hline 386.865 & 155.686 & 912.614 & 300.686 & 87.116 \\
\hline
\end{tabular}

PPD IL-12 


\begin{tabular}{|c|c|c|c|c|}
\hline MEDIUM & $\begin{array}{l}\text { MEDIUM } \\
\text { INFLIX }\end{array}$ & ANTIGEN & $\begin{array}{l}\text { ANTIGEN } \\
\text { INFLIX }\end{array}$ & BEAD \\
\hline & & & & 4488.67 \\
& & & & 2.415 .572 \\
& & & & 651.382 \\
& & & & 399.422 \\
& & & & \\
& & & & 1101.52 \\
& & & & 530.168 \\
& & & 367.71 & 243.594 \\
3.752 .556 & 2730.36 & 1613.28 & 253.659 & 105.522 \\
730.098 & 401.853 & 616.851 & 176.586 & 57.072 \\
& & 386.586 & 291.274 & 174.948 \\
272.694 & 237.784 & 447.228 & 733.066 & 562.988 \\
824.526 & 517.158 & 858.61 & 39.148 & 0 \\
31.848 & 11.556 & 39.148 & 59.32 & 362.322 \\
524.6 & 273.498 & 100.828 & 533.066 & 4488.67 \\
624.526 & 217.158 & 758.61 & & \\
\hline
\end{tabular}

Treated IL-12

\begin{tabular}{|c|c|c|c|c|}
\hline MEDIUM & $\begin{array}{l}\text { MEDIUM } \\
\text { INFLIX }\end{array}$ & ANTIGEN & $\begin{array}{l}\text { ANTIGEN } \\
\text { INFLIX }\end{array}$ & BEAD \\
\hline & & & & 969.03 \\
& & & & 142.626 \\
& & & & 122.706 \\
& & & & 91.84 \\
& & & & 12.399 \\
& & & & 91.84 \\
711.381 & 442.65 & 429.177 & 257.652 & 0 \\
315.468 & 175.278 & 300.522 & 11.939 & 2.580 .028 \\
447.132 & 333.003 & 174.378 & 78.912 & 2.201 .786 \\
94.588 & 128.934 & & & 3.935 .188 \\
0 & 0 & & & 2.691 .226 \\
322.646 & 131.682 & 236.094 & 215.486 & \\
56.12 & 0 & 102.83 & 38.26 & 657.003 \\
56.12 & 0 & & & 1461.35 \\
411.249 & 16.273 & 978.037 & 115.501 & 706.824 \\
1.102 .932 & 634.316 & 414.683 & 428.471 & 4.183 .458 \\
498.703 & 545.51 & 611.824 & 13.534 & 2.497 .922 \\
& & & &
\end{tabular}




\begin{tabular}{|c|c|c|c|c|}
3.212 .822 & 1.719 .089 & 1.852 .769 & 527.589 & 969.03 \\
1.945 .764 & 1.370 .357 & 3.032 .644 & 2.079 .445 & 1.706 .824 \\
711.381 & 442.65 & 429.177 & 257.652 & 1461.35 \\
498.703 & 545.51 & 611.824 & 13.534 & 2.691 .226 \\
1.102 .932 & 634.316 & 434.683 & 428.471 & 2.497 .922 \\
1.945 .764 & 1.370 .357 & 3.032 .644 & 2.079 .445 & 3184.44 \\
\hline
\end{tabular}

Active disease IL-12

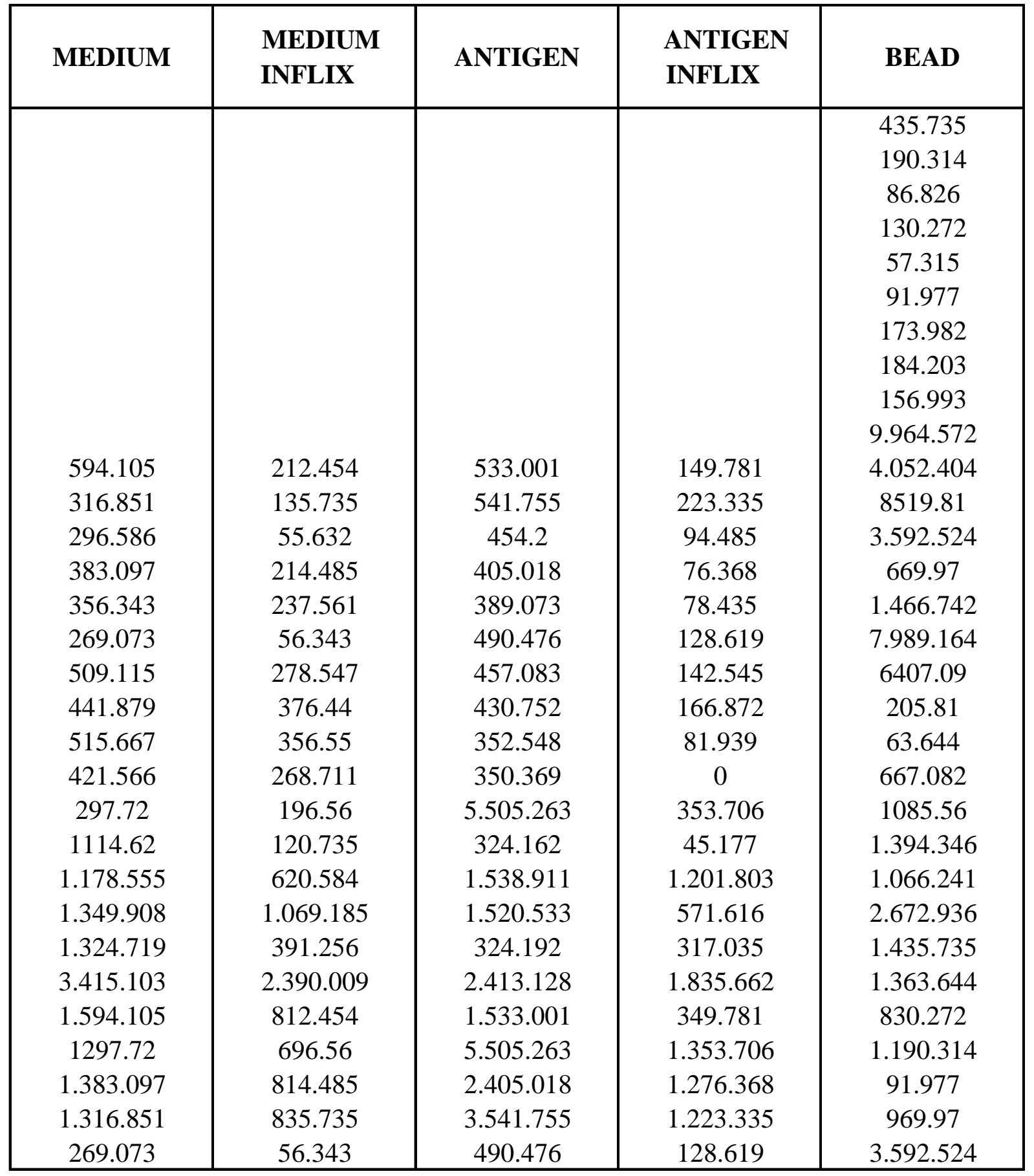

PPD IL- 17 


\begin{tabular}{|c|c|c|c|c|}
\hline MEDIUM & $\begin{array}{l}\text { MEDIUM } \\
\text { INFLIX }\end{array}$ & ANTIGEN & $\begin{array}{l}\text { ANTIGEN } \\
\text { INFLIX }\end{array}$ & BEAD \\
\hline & & & & 207.77 \\
& & & & 257.65 \\
& & & & 138.354 \\
& & & & 238.135 \\
& & & & \\
& & & & 10.258 \\
& & & & 28.189 \\
704.295 & 109.89 & 542.163 & 129.135 & 9.258 \\
171.231 & 36.96 & 122.055 & 54.393 & 43.998 \\
& & 0 & 0 & 80.076 \\
148.13 & 73.654 & 45.896 & 0 & 125.072 \\
164.842 & 0 & 0 & 0 & 123.738 \\
95.02 & 0 & 0 & 0 & 61.424 \\
20.578 & 0 & 0 & 0 & 95.02 \\
44.72 & 0 & 0 & 0 & 29.644 \\
& & & & 207.77 \\
\hline
\end{tabular}

Treated IL-17

\begin{tabular}{|c|c|c|c|c|}
\hline MEDIUM & $\begin{array}{l}\text { MEDIUM } \\
\text { INFLIX }\end{array}$ & ANTIGEN & $\begin{array}{l}\text { ANTIGEN } \\
\text { INFLIX }\end{array}$ & BEAD \\
\hline & & & & 165.231 \\
& & & & 215.751 \\
& & & & 282.018 \\
& & & & 3.844 \\
& & & & 21.532 \\
& & & & 18.294 \\
& & & & 19.912 \\
& & & & 30.494 \\
227.073 & 70.737 & 1.203 .237 & 1.075 .218 & 0 \\
185.046 & 76.071 & 104.046 & 55.794 & 382.5 \\
269.955 & 89.109 & 164.514 & 48.261 & 0 \\
3.048 & 1.456 & & & 7.554 \\
92.788 & 75.606 & & & 0 \\
7.038 & 2.252 & 90.194 & 0 & 5.144 \\
14.258 & 6.238 & 0 & 0 & 877.568 \\
11.042 & 0 & & & 251.024 \\
725.56 & 346.176 & 184.616 & 165.84 & 375.064 \\
539.992 & 232.928 & 269.616 & 99.712 & 343.648 \\
276.4 & 239.52 & 126.248 & 73.88 & 120.608 \\
& & & &
\end{tabular}




\begin{tabular}{|c|c|c|c|c|}
394.376 & 75.632 & 63.472 & 73.184 & 165.231 \\
46.712 & 100.376 & 123.16 & 58.656 & 168.144 \\
227.073 & 70.737 & 1.203 .237 & 975.218 & 375.064 \\
276.4 & 239.52 & 126.248 & 73.88 & 85.144 \\
539.992 & 232.928 & 269.616 & 99.712 & 120.608 \\
346.712 & 100.376 & 123.16 & 58.656 & 23.23 \\
\hline
\end{tabular}

ctive disease IL-17

\begin{tabular}{|c|c|c|c|c|}
\hline MEDIUM & $\begin{array}{l}\text { MEDIUM } \\
\text { INFLIX }\end{array}$ & ANTIGEN & $\begin{array}{c}\text { ANTIGEN } \\
\text { INFLIX }\end{array}$ & BEAD \\
& & & & 213.624 \\
& & & & 353.334 \\
& & & & 394.776 \\
& & & & 800.904 \\
& & & & 272.649 \\
& & & & 201.096 \\
& & & & 160.218 \\
& & & & 225.279 \\
326.565 & 177.564 & 249.135 & 143.823 & 211.041 \\
196.149 & 103.677 & 187.563 & 76.389 & 69.96 \\
292.107 & 122.103 & 103.971 & 83.19 & 162.87 \\
265.275 & 149.643 & 92.697 & 32.046 & 0 \\
191.871 & 91.587 & 38.268 & 4.407 & \\
73.971 & 26.532 & 228.486 & 0 & \\
427.809 & 238.209 & 177.621 & 0 & 100.859 \\
198.663 & 65.769 & 177.954 & 95.199 & 109.548 \\
290.757 & 92.751 & 232.785 & 107.679 & 246.144 \\
156.576 & 141.304 & 141.304 & 91.024 & 91.024 \\
126.248 & 88.144 & 752.992 & 71.056 & 151.648 \\
290.168 & 100.376 & 146.416 & 80.528 & 239.304 \\
130.856 & 75.632 & 120.608 & 110.448 & 178.56 \\
1175.96 & 394.44 & 405.448 & 145.048 & 122.784 \\
269.224 & 215.952 & 114.616 & 98.56 & 213.624 \\
178.56 & 147.88 & 276.472 & 114.616 & 91.024 \\
326.565 & 177.564 & 249.135 & 143.823 & 800.904 \\
126.248 & 88.144 & 752.992 & 71.056 & 953.334 \\
265.275 & 149.643 & 92.697 & 32.046 & 201.096 \\
596.149 & 303.677 & 987.563 & 376.389 & 1.136 .194 \\
73.971 & 26.532 & 228.486 & 0 & 510.859 \\
\hline
\end{tabular}




\begin{tabular}{|c|c|c|}
\hline BEAD INFLIX & $\begin{array}{c}\text { BEAD } \\
\text { ANTIGEN }\end{array}$ & $\begin{array}{c}\text { BCG } \\
\text { ANTIGEN } \\
\text { INFLIX }\end{array}$ \\
\hline 0 & 272.625 & 0 \\
& 142.164 & 0 \\
0 & 53.924 & 0 \\
0 & 31.599 & 28.323 \\
& 520.062 & 15.227 \\
0 & 0 & 0 \\
15.631 & 45.988 & 0 \\
0 & 38.932 & 0 \\
50.496 & 391.632 & 64.992 \\
& 173.487 & 20.358 \\
54.537 & 242.841 & 33.492 \\
17.937 & 96.108 & 56.733 \\
37.05 & 63.09 & 2.841 \\
0 & 36.108 & 24.249 \\
65.928 & 247.743 & 44.418 \\
30.93 & 272.625 & \\
0 & & \\
\hline
\end{tabular}

\begin{tabular}{|c|c|c|}
\hline BEAD INFLIX & $\begin{array}{c}\text { BEAD } \\
\text { ANTIGEN }\end{array}$ & $\begin{array}{c}\text { BCG } \\
\text { ANTIGEN } \\
\text { INFLIX }\end{array}$ \\
\hline 75.252 & 380.187 & 45.246 \\
\hline 12.978 & 37.029 & 0 \\
\hline 37.029 & 0 & 0 \\
\hline 61.923 & & \\
\hline 71.565 & & \\
\hline 76.008 & & \\
\hline 62.4 & 58.155 & 57.687 \\
\hline 59.562 & & \\
\hline 0 & 165.947 & 0 \\
\hline 76.65 & 468.441 & 0 \\
\hline \multirow[t]{2}{*}{50.675} & 290.427 & 0 \\
\hline & & 0 \\
\hline \multirow[t]{2}{*}{48.397} & 687.111 & 78.435 \\
\hline & 0 & 0 \\
\hline 25.684 & 710.423 & 17.513 \\
\hline 0 & 3.222 & 0 \\
\hline 0 & 60.366 & 54.639 \\
\hline 24.49 & 163.362 & 9.918 \\
\hline 45.185 & 89.867 & 75.065 \\
\hline
\end{tabular}




\begin{tabular}{|c|c|c|}
33.298 & 34.714 & 33.883 \\
275.252 & 480.187 & 145.246 \\
54.49 & 363.362 & 19.918 \\
128.36 & 360.366 & 154.639 \\
348.075 & 758.369 & 256.39 \\
93.298 & 432.714 & 233.883 \\
123.6 & 165.947 & 25.369 \\
\hline
\end{tabular}

\begin{tabular}{|c|c|c|}
\hline BEAD INFLIX & $\begin{array}{c}\text { BEAD } \\
\text { ANTIGEN }\end{array}$ & $\begin{array}{c}\text { BCG } \\
\text { ANTIGEN } \\
\text { INFLIX }\end{array}$ \\
\hline 54.42 & 1.898 .079 & 1.622 .358 \\
\hline 112.305 & & \\
\hline 52.989 & 335.538 & 113.958 \\
\hline 133.854 & 76.416 & 70.416 \\
\hline 29.835 & 60.906 & 49.593 \\
\hline 59.871 & 93.552 & 70.416 \\
\hline 77.7 & 49.068 & 0 \\
\hline 0 & 0 & 0 \\
\hline 15.981 & 41.541 & 0 \\
\hline 27.743 & 1.111 .251 & 24.73 \\
\hline 187.518 & 265.022 & 187.518 \\
\hline 30.711 & 553.588 & 25.57 \\
\hline 37.396 & 357.524 & 54.689 \\
\hline 20.953 & 34.173 & 1.593 \\
\hline 0 & 344.697 & 0 \\
\hline 29.096 & 857.513 & 26.471 \\
\hline 29.983 & 152.87 & 30.085 \\
\hline 18.183 & 48.018 & 9.068 \\
\hline 0 & 9.068 & 0 \\
\hline 20.094 & 639.263 & 12.886 \\
\hline 537.806 & 1453.94 & 63.115 \\
\hline 0 & 9.332 & 0 \\
\hline 0 & 0.02 & 0 \\
\hline 0 & 48.09 & 0 \\
\hline 154.42 & 1.898 .079 & 1.522 .358 \\
\hline 89.369 & 399.068 & 123.589 \\
\hline 133.854 & 576.416 & 270.416 \\
\hline 1.112 .305 & & \\
\hline 259.871 & 993.552 & 570.416 \\
\hline 120.953 & 634.173 & 211.593 \\
\hline 237.396 & 357.524 & 154.689 \\
\hline
\end{tabular}




\begin{tabular}{|c|c|c|}
\hline BEAD INFLIX & $\begin{array}{c}\text { BEAD } \\
\text { ANTIGEN }\end{array}$ & $\begin{array}{c}\text { BEAD } \\
\text { ANTIGEN } \\
\text { INFLIX }\end{array}$ \\
\hline 0 & 521.908 & 0 \\
& & \\
0 & 734.291 & 0 \\
0 & 675.926 & 34.992 \\
& 344.637 & 51.958 \\
0 & 0 & 0 \\
121.82 & 40.685 & 0 \\
0 & 0 & 0 \\
0 & 191.94 & 0 \\
107.518 & 297.716 & 85.415 \\
63.753 & 355.956 & 23.457 \\
709.158 & 32.319 & 26.568 \\
856.383 & 61.44 & 130.803 \\
88.188 & 136.989 & 88.188 \\
140.094 & 43.893 & 592.986 \\
46.8 & 38.091 & 46.8 \\
0 & 521.908 & 0 \\
\hline
\end{tabular}

\begin{tabular}{|c|c|c|}
\hline BEAD INFLIX & $\begin{array}{c}\text { BEAD } \\
\text { ANTIGEN }\end{array}$ & $\begin{array}{c}\text { BCG } \\
\text { ANTIGEN } \\
\text { INFLIX }\end{array}$ \\
\hline 135.564 & 219.937 & 0 \\
222.342 & 137.058 & 0 \\
0 & 0 & 0 \\
82.131 & & \\
64.983 & & \\
67.431 & & \\
69.879 & 42.999 & 46.659 \\
58.869 & & \\
0 & 0 & 0 \\
107.261 & 402.083 & 0 \\
0 & 148.544 & 0 \\
0 & 0 & 0 \\
13.401 & & 0 \\
0 & 0 & 21.19 \\
186.516 & 53.516 & 24.094 \\
27.616 & 287.776 & 19.193 \\
17.656 & 28.254 & 16.894 \\
16.762 & 31.376 & 22.163 \\
16.961 & 24.964 & 37.287 \\
42.943 & 358.679 & \\
31.054 & 89.429 &
\end{tabular}




\begin{tabular}{|c|c|c|}
235.564 & 219.937 & 98.326 \\
16.961 & 24.964 & 16.894 \\
16.762 & 31.376 & 19.193 \\
186.516 & 553.516 & 125.36 \\
131.054 & 689.429 & 237.287 \\
23.6 & 123.6 & 45.369 \\
\hline
\end{tabular}

\begin{tabular}{|c|c|c|}
\hline BEAD INFLIX & $\begin{array}{c}\text { BEAD } \\
\text { ANTIGEN }\end{array}$ & $\begin{array}{c}\text { BCG } \\
\text { ANTIGEN } \\
\text { INFLIX } \\
\end{array}$ \\
\hline 0 & 0 & 0 \\
\hline 0 & 0 & 0 \\
\hline 0 & 0 & 0 \\
\hline \multirow{3}{*}{$\begin{array}{c}45.621 \\
106.281\end{array}$} & 1.772 .271 & 1.309 .257 \\
\hline & & \\
\hline & $\begin{array}{c}83.178 \\
123.555\end{array}$ & $\begin{array}{l}50.859 \\
82995\end{array}$ \\
\hline 360.675 & 123.555 & 82.995 \\
\hline 159.537 & 10.677 & 0 \\
\hline 0 & 0 & 0 \\
\hline 20.152 & 295.193 & 0 \\
\hline 0 & 0 & 0 \\
\hline 0 & 0 & 0 \\
\hline 0 & 0 & 0 \\
\hline 40.44 & 32.446 & 0 \\
\hline 22.164 & 16.517 & 0 \\
\hline 0 & 513.166 & 0 \\
\hline 0 & 0 & 0 \\
\hline 15.715 & 18.712 & 15.658 \\
\hline 15.949 & 18.791 & 15.658 \\
\hline 28.116 & 65.136 & 51.176 \\
\hline 21.638 & 149.961 & 38.386 \\
\hline 61.356 & 220.647 & 16.277 \\
\hline 47.976 & 143.667 & 22.53 \\
\hline 534.708 & 152.024 & 59.421 \\
\hline 89.369 & 256.369 & 123.369 \\
\hline 115.949 & 358.791 & 215.658 \\
\hline 145.621 & 2.772 .271 & 1.309 .257 \\
\hline 493.369 & 2.893 .369 & 1.589 .363 \\
\hline 944.918 & 1.883 .178 & 950.859 \\
\hline 40.44 & 132.446 & 23.693 \\
\hline 23.369 & 248.369 & 112.699 \\
\hline
\end{tabular}




\begin{tabular}{|c|c|c|}
\hline BEAD INFLIX & $\begin{array}{c}\text { BEAD } \\
\text { ANTIGEN }\end{array}$ & $\begin{array}{c}\text { BCG } \\
\text { ANTIGEN } \\
\text { INFLIX }\end{array}$ \\
\hline 608.544 & 223.163 & 652.218 \\
213.491 & 2.507 .358 & 289.898 \\
2.794 .734 & 850.198 & 2.297 .778 \\
30.941 & 210.09 & 182.265 \\
& 674.988 & 699.576 \\
0 & 0 & 0 \\
28.789 & 47.814 & 183.522 \\
16.479 & 8.112 & 0 \\
812.994 & 1.355 .115 & 2.255 .964 \\
436.884 & 455.907 & 465.45 \\
39.639 & 87.723 & 50.763 \\
41.052 & 37.509 & 30.06 \\
43.002 & 153.108 & 27.108 \\
30.6 & 69.759 & 36.09 \\
36.069 & 369.78 & 3.09 \\
60.522 & 266.121 & 36.09 \\
308.544 & 623.163 & 252.218 \\
\hline
\end{tabular}

\begin{tabular}{|c|c|c|}
\hline BEAD INFLIX & $\begin{array}{c}\text { BEAD } \\
\text { ANTIGEN }\end{array}$ & $\begin{array}{c}\text { BCG } \\
\text { ANTIGEN } \\
\text { INFLIX }\end{array}$ \\
\hline 436.884 & & \\
7.167 & 17.292 & 9.981 \\
7.167 & 3.423 & 0 \\
56.12 & & \\
24.522 & & \\
78.102 & & \\
4.705 & 9.513 & 0 \\
0 & & \\
28.269 & 33.171 & 55.484 \\
271.023 & 365.15 & 246.512 \\
540.275 & 934.91 & 711.063 \\
522.633 & 369.422 & 159.161 \\
75.696 & 61.221 & 0 \\
9.337 & 5.86 & 13.961 \\
308.945 & 80.05 & 0 \\
472.106 & 702.44 & 417.856 \\
35.312 & 56.508 & 48.188 \\
33.524 & 62.752 & 38.386 \\
33.922 & 49.928 & 33.788 \\
85.886 & 717.358 & 44.326 \\
62.108 & 178.858 & 74.574
\end{tabular}




\begin{tabular}{|c|c|c|}
436.884 & & \\
323.922 & 1.249 .928 & 523.788 \\
53.524 & 262.752 & 68.386 \\
308.945 & 580.05 & 189 \\
262.108 & 178.858 & 74.574 \\
28.269 & 133.171 & 55.484 \\
\hline
\end{tabular}

\begin{tabular}{|c|c|c|}
\hline BEAD INFLIX & $\begin{array}{c}\text { BEAD } \\
\text { ANTIGEN }\end{array}$ & $\begin{array}{c}\text { BCG } \\
\text { ANTIGEN } \\
\text { INFLIX }\end{array}$ \\
\hline 812.994 & 1.321 .074 & 1.803 .027 \\
780.792 & 4.413 .128 & 2.410 .399 \\
1.007 .073 & 1.320 .933 & 788.598 \\
0 & 0 & 0 \\
0 & 0 & 0 \\
0 & 0 & 0 \\
31.722 & 27.921 & 16.566 \\
16.566 & 5.292 & 6.231 \\
24.129 & 26.022 & 11.859 \\
759.933 & 1010.44 & 640.642 \\
154.602 & 92.736 & 179.31 \\
421.403 & 396.542 & 244.887 \\
5.666 & 22.827 & 14.017 \\
192.533 & 243.514 & 104.148 \\
660.988 & 950.269 & 822.474 \\
31.754 & 419.061 & 33.646 \\
157.676 & 436.171 & 313.116 \\
31.43 & 37.424 & 31.316 \\
31.898 & 37.582 & 31.316 \\
56.232 & 130.272 & 102.352 \\
43.276 & 299.922 & 76.772 \\
122.712 & 441.294 & 32.554 \\
95.952 & 287.334 & 45.06 \\
1.069 .416 & 304.048 & 118.842 \\
812.994 & 1.821 .074 & 1.303 .027 \\
231.898 & 837.582 & 331.316 \\
125.369 & 689.36 & 258.369 \\
1.280 .792 & 4.413 .128 & 2.410 .399 \\
231.722 & 927.921 & 216.566 \\
392.533 & 543.514 & 294.148 \\
25.666 & 122.827 & 54.017 \\
\hline & &
\end{tabular}




\begin{tabular}{|c|c|c|}
\hline BEAD INFLIX & $\begin{array}{c}\text { BEAD } \\
\text { ANTIGEN }\end{array}$ & $\begin{array}{c}\text { BCG } \\
\text { ANTIGEN } \\
\text { INFLIX }\end{array}$ \\
\hline 1.249 .246 & 3.219 .046 & 0 \\
1.488 .906 & 2371.25 & 1329.39 \\
0 & 559.624 & 0 \\
137.3 & 441.568 & 163.816 \\
& 2.822 .266 & 835.494 \\
1.236 .966 & 901.222 & 905.972 \\
317.344 & 665.562 & 232.676 \\
447.648 & 694.328 & 523.886 \\
135.735 & 1.129 .956 & 682.86 \\
69.03 & 432.435 & 284.142 \\
54.471 & 414.243 & 263.568 \\
122.88 & 143.154 & 123.598 \\
318.332 & 600.204 & 482.328 \\
7.678 & 0 & 0 \\
18.71 & 12.204 & 28.546 \\
118.332 & 200.204 & 182.328 \\
1.249 .246 & 3.219 .046 & 985.3 \\
\hline
\end{tabular}

\begin{tabular}{|c|c|c|}
\hline BEAD INFLIX & $\begin{array}{c}\text { BEAD } \\
\text { ANTIGEN }\end{array}$ & $\begin{array}{c}\text { BCG } \\
\text { ANTIGEN } \\
\text { INFLIX }\end{array}$ \\
\hline 600.249 & 1.427 .613 & 781.623 \\
71.733 & 359.16 & 270.522 \\
34.248 & 254.709 & 208.056 \\
56.12 & & \\
24.522 & & \\
78.102 & & \\
9.41 & 19.026 & 0 \\
& & \\
1.801 .428 & 6.475 .802 & 1.670 .322 \\
3.048 .956 & 2.258 .498 & 2.657 .434 \\
503.172 & 1358.72 & 710.472 \\
1493.54 & 3.924 .818 & 1171.06 \\
273.646 & 1.149 .536 & 391.478 \\
2.271 .714 & 5.215 .826 & 1.843 .488 \\
1.255 .206 & & \\
& & 115.501 \\
152.358 & 2.148 .136 & 215.107 \\
262.324 & 1147.05 & 297.72 \\
262.324 & 377.211 & 1.050 .686 \\
1.713 .276 & 2.137 .566 & 1.358 .733 \\
2.265 .435 & 1695.84 &
\end{tabular}




\begin{tabular}{|c|c|c|}
600.249 & 1.427 .613 & 781.623 \\
562.324 & 877.211 & 297.72 \\
262.324 & 1147.05 & 215.107 \\
1.255 .206 & 3.703 .686 & 1.236 .023 \\
2.265 .435 & 1695.84 & 1.358 .733 \\
1.801 .428 & 6.475 .802 & 1.670 .322 \\
\hline
\end{tabular}

\begin{tabular}{|c|c|c|}
\hline BEAD INFLIX & $\begin{array}{c}\text { BEAD } \\
\text { ANTIGEN }\end{array}$ & $\begin{array}{c}\text { BCG } \\
\text { ANTIGEN } \\
\text { INFLIX }\end{array}$ \\
\hline 269.697 & 824.097 & 306.468 \\
144.825 & 1009.17 & 736.851 \\
60.9 & 376.029 & 139.908 \\
81.276 & 365.058 & 65.058 \\
55.632 & 295.866 & 60.108 \\
62.523 & 370.791 & 245.058 \\
92.862 & 297.201 & 171.366 \\
62.442 & 281.154 & 58.056 \\
35.496 & 294.003 & 41.133 \\
9.066 .564 & 10294.63 & 9.135 .216 \\
1.573 .724 & 3883.24 & 1.318 .668 \\
4.406 .594 & 8161.98 & 5.224 .554 \\
1.064 .764 & 3.301 .188 & 726.506 \\
775.46 & 538.568 & 383.56 \\
425.426 & 1.002 .408 & 323.108 \\
4.399 .774 & 8.356 .878 & 3.253 .738 \\
4.052 .404 & 6.718 .136 & 2.907 .662 \\
184.304 & 199.638 & 130.215 \\
10.802 & 114.519 & 72.166 \\
48.236 & 1114.62 & 125.36 \\
922.818 & 1.951 .576 & 539.213 \\
589.334 & 1.237 .724 & 653.54 \\
462.846 & 324.035 & 189.023 \\
1.230 .516 & 1.842 .659 & 365.529 \\
969.697 & 1.824 .097 & 1.206 .468 \\
510.802 & 2.114 .519 & 772.166 \\
381.276 & 1.365 .058 & 765.058 \\
944.825 & 3009.17 & 1.736 .851 \\
62.523 & 370.791 & 245.058 \\
775.46 & 1.538 .568 & 883.56 \\
1.064 .764 & 3.301 .188 & 726.506 \\
\hline
\end{tabular}




\begin{tabular}{|c|c|c|}
\hline BEAD INFLIX & $\begin{array}{c}\text { BEAD } \\
\text { ANTIGEN }\end{array}$ & $\begin{array}{c}\text { BCG } \\
\text { ANTIGEN } \\
\text { INFLIX }\end{array}$ \\
\hline 0 & 0 & 0 \\
0 & 0 & 0 \\
0 & 0 & 0 \\
0 & 1247.19 & 781.044 \\
& 15.712 & 6.086 \\
0 & 208.343 & 114.783 \\
85.585 & 1.449 .388 & 233.202 \\
0 & 400.584 & 77.147 \\
150.663 & 169.143 & 43.998 \\
34.998 & 138.108 & 34.986 \\
34.833 & 350.667 & 0 \\
0 & 0 & 0 \\
0 & 0 & 0 \\
0 & 0 & 0 \\
0 & 0 & 0 \\
0 & 0 & 0 \\
102.362 & 89.32 & 23.06 \\
\hline
\end{tabular}

\begin{tabular}{|c|c|c|}
\hline BEAD INFLIX & $\begin{array}{c}\text { BEAD } \\
\text { ANTIGEN }\end{array}$ & $\begin{array}{c}\text { BCG } \\
\text { ANTIGEN } \\
\text { INFLIX }\end{array}$ \\
\hline 37.953 & 799.83 & 461.769 \\
57.981 & 81.063 & 38.868 \\
87.906 & 66.897 & 19.167 \\
0 & & \\
3.844 & & \\
10.24 & & \\
1.456 & 2.252 & 0 \\
24.782 & & \\
0 & 0 & 0 \\
6.054 & 5.866 & 0 \\
23.086 & 15.206 & 0 \\
8.07 & 5.978 & 0 \\
442.82 & 6.008 & 0 \\
121.316 & 283.797 & 0 \\
5.104 & 13.53 & 9.582 \\
5.222 & 0 & 0 \\
178.32 & 181.464 & 79.64 \\
293.512 & 307.36 & 181.464 \\
120.288 & 328.4 & 102.624 \\
128.288 & 767.848 & 78.08 \\
63.472 & 566.392 & 46.712 \\
& &
\end{tabular}




\begin{tabular}{|c|c|c|}
37.953 & 799.83 & 461.769 \\
120.288 & 328.4 & 102.624 \\
293.512 & 307.36 & 181.464 \\
25.104 & 113.53 & 39.582 \\
63.472 & 566.392 & 246.712 \\
0 & 36.236 & 0 \\
\hline
\end{tabular}

\begin{tabular}{|c|c|c|}
\hline BEAD INFLIX & $\begin{array}{c}\text { BEAD } \\
\text { ANTIGEN }\end{array}$ & $\begin{array}{c}\text { BCG } \\
\text { ANTIGEN } \\
\text { INFLIX }\end{array}$ \\
\hline 123.915 & 93.942 & 70.737 \\
62.997 & 94.005 & 54.393 \\
105.54 & 30 & 14.856 \\
367.245 & 117.639 & 25.62 \\
62.811 & 31.848 & 0 \\
68.829 & 0 & 0 \\
92.751 & 59.082 & 40.926 \\
52.539 & 51.465 & 29.802 \\
77.238 & 77.238 & 43.002 \\
0 & 0 & 0 \\
0 & 0 & 0 \\
0 & 0 & 0 \\
127.062 & 71.247 & 104.446 \\
& 459.569 & 61.396 \\
05.96 & 511.144 \\
0 & 9.92 & 0 \\
0 & 0 & 0 \\
120.288 & 328.144 & 226.368 \\
65.432 & 187.776 & 91.024 \\
73.184 & 712.072 & 146.416 \\
65.896 & 136.024 & 46.712 \\
98.56 & 188.632 & 80.328 \\
123.915 & 114.616 & 88.08 \\
65.432 & 111.912 & 62.616 \\
367.245 & 187.776 & 70.737 \\
562.997 & 1.117 .639 & 91.024 \\
68.829 & 358.369 & 825.62 \\
997.236 & 371.247 & 854.393 \\
127.062 & & 258.398 \\
& & 104.446 \\
\hline
\end{tabular}

